\title{
Structural, functional, and evolutionary analysis of Cry toxins of Bacillus thuringiensis: an in silico study
}

\author{
Sujit Kumar Das ${ }^{1,2}$, Sukanta Kumar Pradhan ${ }^{1}$, Kailash Chandra Samal ${ }^{3}$ and Nihar Ranjan Singh ${ }^{4 *}$
}

\begin{abstract}
Background: Bacillus thuringiensis (Bt) is a gram-positive spore-forming soil bacterium that synthesizes crystalline (Cry) protein, which is toxic and causing pathogenicity against mainly three insect orders: Coleoptera, Diptera, and Lepidoptera. These crystalline protein inclusions, i.e., $\delta$-endotoxins are successfully used as a bio-control agent against insect pests.

Main body: A total of 58 various Cry proteins belonging to these 3 insect orders were retrieved from SwissProt database and are categorized into different groups. Structural and functional analysis were performed to understand the functional domain arrangements at sequence level as well as at structural level involving both experimental and predicted 3-dimensional models. Besides, the analysis of evolutionary relationship involving all 58 observed Cry proteins at the sequence, domain, and structural levels were done using different bioinformatics tools. Evolutionary analysis revealed that some Cry proteins having toxicity for a specific insect order are found to be clustered for another different insect order, which concludes that they might have toxicity for more than one insect order. Threedimensional (3D) structure analysis of both experimental and predicted models revealed that proteins might have toxicity for a specific insect order differ in their structural arrangements and was observed in Cry proteins belonging to 3 different insect orders.

Conclusions: It could be hypothesized that an inner-molecular domain shift or domain insertion/deletion might have taken place during the evolutionary process, which consequently causes structural and functional divergence of $B t$. The study output may be helpful for understanding the diversity as well as specificity of the analyzed insecticidal proteins and their application as a biopesticide in the field of agriculture.
\end{abstract}

Keywords: Bacillus thuringiensis, Biopesticides, Cry toxins, Bioinformatics, Phylogenetic analysis

\section{Background}

Bacillus thuringiensis $(B t)$ is a gram-positive sporeforming soil bacterium causing pathogenicity in insects. $B t$ strains synthesize Crystal (Cry) and cytolytic (Cyt) toxins (also known as $\delta$-endotoxins) that have a natural insecticidal effect on selective insect orders. $\delta$-endotoxins of $B t$ are being used successfully as a biological control agent against some insect pests (Schnepf 1995). These

\footnotetext{
* Correspondence: nihar.singh@gmail.com

${ }^{4}$ Department of Botany and Center of Excellence in Environment and Public Health, Ravenshaw University, Cuttack, Odisha 753003, India

Full list of author information is available at the end of the article
}

endotoxins are exclusively active against larval stages of different insect orders such as Lepidoptera (Butterflies, Moths), Coleoptera (Flies and Mosquitoes), and Diptera (Beetles and Weevils) (Raymond et al. 2010). During sporulation phase, $B t$ produces these Cry or Cyt toxins that have hazardous effect on insects (Bravo et al. 2011). Cry and Cyt toxins are considered as parasporal inclusion proteins from $B t$ that exhibit toxic effects and hemolytic activity respectively (Crickmore et al. 1998).These two types of toxins belong to a class of pore-forming toxins (PFTs) that are secreted as water-soluble proteins and undergo conformational changes in order to insert into
Springer Open (c) The Author(s). 2021 Open Access This article is licensed under a Creative Commons Attribution 4.0 International License, which permits use, sharing, adaptation, distribution and reproduction in any medium or format, as long as you give appropriate credit to the original author(s) and the source, provide a link to the Creative Commons licence, and indicate if changes were made. The images or other third party material in this article are included in the article's Creative Commons licence, unless indicated otherwise in a credit line to the material. If material is not included in the article's Creative Commons licence and your intended use is not permitted by statutory regulation or exceeds the permitted use, you will need to obtain permission directly from the copyright holder. To view a copy of this licence, visit http://creativecommons.org/licenses/by/4.0/. 
the host membrane. When Crystal (Cry) toxins ingested by insects are get solubilized in their midgut, then it gets proteolytically activated by midgut proteases and bind to specific receptors located in the insect cell membrane leading to cell disruption and cell death (Bravo et al. 2007). Most Cry proteins exist as inactive protoxins that can be converted into active toxins by certain kinds of insect midgut proteases (Höfte and Whiteley 1989). This activation process appears to involve a sequential series of proteolytic cleavages, starting at the C-terminus and proceeding toward the $\mathrm{N}$-terminus until the protease-stable toxin is generated (Choma and Kaplan 1990). Besides these membrane proteins, other components have been identified due to their capacity to interact with 3d-Cry toxins (3 domain-Cry toxins) such as glycolipids, intracellular proteins, V-ATPase subunit A or actin (McNall and Adang 2003; Griffitts et al. 2005; Bayyareddy et al. 2009). The interaction of Cry1 toxins with different proteins present in lepidopteran midgut cells is a complex process involving multiple membrane proteins such as cadherinlike proteins (CADs), aminopeptidase N (APN), and alkaline phosphatase (ALP) (Pigott and Ellar 2007; Soberon et al. 2009).

Extensive screening of $B t$ strains and Cry gene sequencing has led to the identification of more than 700 Cry gene sequences (Höfte and Whiteley 1989; Van Frankenhuyzen 2009; Crickmore et al. 2011). These sequences have been classified according to their amino acid sequence identities in 70 different Cry gene groups (Cry1, Cry2...Cry 70) where toxins belonging to each group share less than $40 \%$ amino acid identity with proteins from other groups (Crickmore et al. 1998). Within each group, a capital letter (Cry1A, Cry1B, etc.) is given when they share less than 70\% identity. A small letter (Cry1Aa, Cry1 $\mathrm{Ab}$, etc.) is given when toxins share more than $70 \%$ but less than 95\% identity (Bravo et al. 2013). Many Cry proteins show pesticidal properties, while some protein produced as parasporal crystals that have unknown invertebrate targets called as parasporins such as Cry31A, Cry41A, Cry45A, Cry46A, Cry63A, and Cr64A exhibit strong and specific cytocidal activity against human cancer cell (Mizuki et al. 2000). Phylogenetic analysis of Cry toxins shows that the great variability in the biocidal activity of the $3 \mathrm{~d}$-Cry group has resulted from the independent evolution of the 3 structural domains and the domain III swapping among different toxins. These two processes have generated proteins with similar modes of action but with different specificities (de Maagd et al. 2001). Various 3-dimensional crystal structures of activated Cry toxins have been determined by experimental methods. These are Coleoptera-specific Cry3Aa (PDB entry: 1DLC) (Li et al. 1991) and Cry3Bb1 (PDB entry: 1JI6) (Galitsky et al. 2001), Cry 34 Ab1(PDB entry: 4JOX) and Cry 35 Ab1 (PDB entry: 4JPo) (Kelker et al.
2014), Lepidoptera-specific Cry1Aa (PDB entry: 1CIY) (Grochulski et al. 1995), Cry1Ac (PDB entry: 4ARX/ 4ARY/4W8J) (Evdokimov et al. 2014), Cry1Da (PDB entry: 6OVB) (Wang et al. 2019), Cry1Fa (PDB entry: 6DJ4) (Wang et al. 2018), Cry1Be (PDB entry: 6OWK) (Wang et al. 2019), Lepidoptera/Diptera-specific Cry2Aa (PDB entry: 1I5P) (Morse et al. 2001), Diptera-specific Cry4-Ba (PDB entry: 1W99) (Boonserm et al. 2005), and Cry4Aa (PDB entry: 2C9K) (Boonserm et al. 2006). Although these toxins exhibit markedly different insecticidal specificities, the overall folding patterns of their structures are quite similar, comprising 3 domains. The domain (I) is a bundle of 7-8 $\alpha$ helices involved in pore formation, domain (II) is a $\beta$-prism with exposed loops regions involved in receptor binding, and domain (III) is a $\beta$-sandwich and has influence on receptor binding, ion channel formation, and insect specificity ( $\mathrm{Li}$ et al. 1991; Grochulski et al. 1995; Derbyshire et al. 2001; Galitsky et al. 2001; Morse et al. 2001; Guo et al. 2009, and Hui et al. 2012).

It has been observed that the Crystal toxins showed specificity to different insect orders which signify that they might have shared certain level of relationships at different levels such as sequence, domain, and structure. The present article aimed to retrieve different Cry proteins from various protein databases, followed by phylogenetic analysis, domain characterization, structure predictions, experimental structure analysis, and comparison.

\section{Main text}

\section{Sequence retrieval and analysis of Cry proteins}

Crystal (Cry) proteins of $B t$ having specificity to 3 broad insect orders: Lepidoptera, Coleoptera, and Diptera were retrieved from the UniprotKB protein sequence database (https://www.uniprot.org/). Selections were made only from manually annotated and reviewed sequences belonging to Swiss-Prot and stored in FASTA format for further bioinformatics analysis.

\section{Domain identification, characterization, and functional analysis}

Identification and annotation of genetically mobile domains and its architectures in the manually annotated and reviewed protein sequences were carried out using a web-based bioinformatics domain prediction tool, i.e., NCBI Batch CD-Search service (https://www.ncbi.nlm. nih.gov/Structure/bwrpsb/bwrpsb.cgi). These domains are extensively annotated with respect to phyletic distributions, functional class, tertiary structures, and functionally important residues.

\section{Sequence alignment and phylogenetic tree construction} Divergence analysis among the retrieved Crystal proteins and the predicted domains were conducted in Molecular 
Table 1 List of cry proteins having toxicity for Coleoptera insect order

\begin{tabular}{lllllll}
\hline Entry & Protein & Gene & Organism & Length & PDB ID & Insect_Order \\
\hline P17969 & cry3Ba & cry3Ba & Bacillus thuringiensis subsp. tolworthi & 659 & N/A & Coleoptera \\
P0A379 & cry3Aa & cry3Aa & Bacillus thuringiensis subsp. tenebrionis & 644 & 1DLC,4QX0,4QX1,4QX2,4QX3 & Coleoptera \\
P0A381 & cry3Aa & cry3Aa & Bacillus thuringiensis subsp. san diego & 644 & N/A & Coleoptera \\
P0A380 & cry3Aa & cry3Aa & Bacillus thuringiensis subsp. morrisoni & 644 & N/A & Coleoptera \\
Q45744 & cry3Ca & cry3Ca & Bacillus thuringiensis subsp. kurstaki & 649 & N/A & Coleoptera \\
Q45704 & cry8Aa & cry8Aa & Bacillus thuringiensis subsp. kumamotoensis & 1157 & N/A & Coleoptera \\
Q45705 & cry8Ba & cry8Ba & Bacillus thuringiensis subsp. kumamotoensis & 1169 & N/A & Coleoptera \\
Q45708 & cry7Ab & cry7Ab & Bacillus thuringiensis subsp. kumamotoensis & 1138 & N/A & Coleoptera \\
Q45706 & cry8Ca & cry8Ca & Bacillus thuringiensis subsp. japonensis & 1160 & N/A & Coleoptera \\
O06014 & cry9Da & cry9Da & Bacillus thuringiensis subsp. japonensis & 1169 & N/A & Coleoptera \\
Q45707 & cry7Ab & cry7Ab & Bacillus thuringiensis subsp. dakota & 1138 & N/A \\
Q939T0 & cry34Ab1 & N/A & Bacillus thuringiensis & 123 & $4 J O X$ & Coleoptera \\
Q03749 & cry7Aa & cry7Aa & Bacillus thuringiensis & 1138 & N/A & Coleoptera \\
Q939S9 & cry35Ab1 & N/A & Bacillus thuringiensis & 383 & $4 J P 0$ & Coleoptera \\
Q06117 & cry3Bb1 & cry3Bb1 & Bacillus thuringiensis & 652 & 1Jl6 & Coleoptera \\
\hline
\end{tabular}

Evolutionary Genetics Analysis X (MEGA X) (Kumar et al. 2018) using Neighbor-Joining (NJ) method (Saitou and Nei 1987). The predicted phylogenetic tree was evaluated using bootstrap reliability test for 1000 replicates. The tree was drawn to scale, with branch lengths in the same units as those of the evolutionary distances used to infer the phylogenetic tree. The evolutionary distances were computed using the Poisson correction method (Zuckerkandl and Pauling 1965) and are in the units of the number of amino acid substitutions per site.

\section{Structural diversity analysis and prediction}

The selected Cry proteins belonging to the 3 major insect orders Lepidoptera, Coleoptera, and Diptera were searched in RCSB-PDB (Research Collaborator for Structural Bioinformatics-Protein Data Bank) (https://www. rcsb.org/) for availability of experimentally solved 3dimensional structures and PDB files were downloaded.
The protein sequences having no 3-dimensional structures in PDB were submitted to web based Protein Homology/analogY Recognition Engine V2.0 (Phyre ${ }^{2}$ ) (http:// www.sbg.bio.ic.ac.uk/ phyre2/html/page.cgi?id=index) for protein modeling and prediction of 3-dimensional structures.

\section{Crystal (Cry) proteins of Bacillus thuringiensis}

Total 58 Cry protein sequences of bacteria $B t$ were retrieved from UniProtKB database (only annotated and reviewed). Among all of these 58 sequences, total 15, 10, and 33 numbers of Cry proteins were observed in the insect orders: Coleoptera, Diptera, and Lepidoptera, respectively. Primary sequence variation in length of Cry proteins were noticed as 123-1169 amino acids in case of order Coleoptera (Table 1), 643-1180 amino acids in Diptera (Table 2), and mostly more than 1100 amino acids in Lepidoptera (Table 3).

Table 2 List of cry proteins having toxicity for Diptera insect order

\begin{tabular}{|c|c|c|c|c|c|c|}
\hline Entry & Protein & Gene & Organism & Length & PDB ID & Insect_Order \\
\hline Q9ZIU5 & cry $11 \mathrm{Bb}$ & cry $11 \mathrm{Bb}$ & Bacillus thuringiensis subsp. medellin & 750 & N/A & Diptera \\
\hline O32307 & cry19Aa & cry19Aa & Bacillus thuringiensis subsp. Jegathesan & 648 & $N / A$ & Diptera \\
\hline Q45730 & cry11Ba & cry11Ba & Bacillus thuringiensis subsp. Jegathesan & 724 & $N / A$ & Diptera \\
\hline P05519 & cry4Ba & cry4Ba & Bacillus thuringiensis subsp. Israelensis & 1136 & 1W99,4MOA & Diptera \\
\hline P09662 & cry10Aa & cry10Aa & Bacillus thuringiensis subsp. Israelensis & 675 & $N / A$ & Diptera \\
\hline P16480 & cry4Aa & cry4Aa & Bacillus thuringiensis subsp. Israelensis & 1180 & $2 \mathrm{C} 9 \mathrm{~K}$ & Diptera \\
\hline P21256 & cry11Aa & cry11Aa & Bacillus thuringiensis subsp. Israelensis & 643 & $N / A$ & Diptera \\
\hline Q9S597 & cry27Aa & cry27Aa & Bacillus thuringiensis subsp. higo & 826 & $N / A$ & Diptera \\
\hline О86170 & cry19Ba & cry19Ba & Bacillus thuringiensis subsp. higo & 682 & $N / A$ & Diptera \\
\hline O32321 & cry20Aa & cry20Aa & Bacillus thuringiensis subsp. fukuokaensis & 753 & $N / A$ & Diptera \\
\hline
\end{tabular}


Table 3 List of cry proteins having toxicity for Lepidoptera insect order

\begin{tabular}{|c|c|c|c|c|c|c|}
\hline Entry & Protein & Gene & Organism & Length & PDB ID & Insect_Order \\
\hline Q9ZAZ5 & cry $1 \mathrm{Bd}$ & cry $1 \mathrm{Bd}$ & Bacillus thuringiensis subsp. wuhanensis & 1231 & N/A & lepidoptera \\
\hline Q9ZAZ6 & cry1Gb & cry1Gb & Bacillus thuringiensis subsp. wuhanensis & 1169 & N/A & lepidoptera \\
\hline Q45733 & cry9Ca & cry9Ca & Bacillus thuringiensis subsp. tolworthi & 1157 & N/A & lepidoptera \\
\hline Q45729 & cry15Aa & cry $15 \mathrm{Aa}$ & Bacillus thuringiensis subsp. thompsoni & 340 & N/A & lepidoptera \\
\hline P0A369 & cry1Aa & cry1Aa & Bacillus thuringiensis subsp. sotto & 934 & N/A & lepidoptera \\
\hline Q45715 & cry1Ka & cry1Ka & Bacillus thuringiensis subsp. morrisoni & 1215 & N/A & lepidoptera \\
\hline Q45718 & cry1Hb & cry $1 \mathrm{Hb}$ & Bacillus thuringiensis subsp. morrisoni & 1155 & N/A & lepidoptera \\
\hline O66377 & cry1Fb & cry1Fb & Bacillus thuringiensis subsp. morrisoni & 1169 & $N / A$ & lepidoptera \\
\hline P0A370 & cry $1 \mathrm{Ab}$ & cry $1 \mathrm{Ab}$ & Bacillus thuringiensis subsp. kurstaki & 1155 & N/A & lepidoptera \\
\hline P0A366 & cry1Aa & cry1Aa & Bacillus thuringiensis subsp. kurstaki & 1176 & $1 \mathrm{ClY}$ & lepidoptera \\
\hline P21254 & cry $2 \mathrm{Ab}$ & cry $2 \mathrm{Ab}$ & Bacillus thuringiensis subsp. kurstaki & 633 & N/A & lepidoptera \\
\hline P05068 & cry $1 \mathrm{Ac}$ & cry $1 \mathrm{Ac}$ & Bacillus thuringiensis subsp. kurstaki & 1178 & 4ARX, 4ARY,4W8J & lepidoptera \\
\hline Q57458 & cry1Ea & cry1Ea & Bacillus thuringiensis subsp. kenyae & 1171 & N/A & lepidoptera \\
\hline Q99031 & cry9Aa & cry9Aa & Bacillus thuringiensis subsp. galleriae & 1156 & N/A & lepidoptera \\
\hline P0A368 & cry $1 \mathrm{Aa}$ & cry1Aa & Bacillus thuringiensis subsp. entomocidus & 1176 & N/A & lepidoptera \\
\hline P0A375 & cry $1 \mathrm{Ca}$ & cry $1 \mathrm{Ca}$ & Bacillus thuringiensis subsp. entomocidus & 1189 & N/A & lepidoptera \\
\hline Q03748 & cry $1 \mathrm{Ae}$ & cry $1 \mathrm{Ae}$ & Bacillus thuringiensis subsp. alesti & 1181 & N/A & lepidoptera \\
\hline P0A376 & cry $1 \mathrm{Ca}$ & cry1Ca & Bacillus thuringiensis subsp. aizawai & 1189 & N/A & lepidoptera \\
\hline P0A367 & cry $1 \mathrm{Aa}$ & cry1Aa & Bacillus thuringiensis subsp. aizawai & 1176 & N/A & lepidoptera \\
\hline Q03744 & cry1Ad & cry1Ad & Bacillus thuringiensis subsp. aizawai & 1179 & N/A & lepidoptera \\
\hline P19415 & cry1Da & cry1Da & Bacillus thuringiensis subsp. aizawai & 1165 & $6 \mathrm{OVB}$ & lepidoptera \\
\hline Q03745 & cry1Eb & cry1Eb & Bacillus thuringiensis subsp. aizawai & 1174 & N/A & lepidoptera \\
\hline Q03746 & cry1Fa & cry1Fa & Bacillus thuringiensis subsp. aizawai & 1174 & $6 \mathrm{DJ} 4$ & lepidoptera \\
\hline Q9ZNL9 & cry9Ea & cry9Ea & Bacillus thuringiensis subsp. aizawai & 1150 & N/A & lepidoptera \\
\hline Q45746 & cry $1 \mathrm{Ga}$ & cry $1 \mathrm{Ga}$ & Bacillus thuringiensis & 1166 & N/A & Lepidoptera \\
\hline Q9XDL1 & crylld & crylld & Bacillus thuringiensis & 719 & N/A & Lepidoptera \\
\hline O85805 & cry1Be & cry1Be & Bacillus thuringiensis & 1227 & $60 W K$ & Lepidoptera \\
\hline Q45748 & cry1Ha & cry $1 \mathrm{Ha}$ & Bacillus thuringiensis & 1172 & N/A & Lepidoptera \\
\hline Q45716 & cry1 Jb & cry1Jb & Bacillus thuringiensis & 1170 & N/A & Lepidoptera \\
\hline Q9S515 & cry $1 \mathrm{Ag}$ & cry $1 \mathrm{Ag}$ & Bacillus thuringiensis & 1176 & N/A & Lepidoptera \\
\hline Q45747 & cry1Db & cry1Db & Bacillus thuringiensis & 1160 & N/A & Lepidoptera \\
\hline Q45738 & cry1Ja & cry1Ja & Bacillus thuringiensis & 1167 & N/A & Lepidoptera \\
\hline Q45739 & cry1Bb & cry1Bb & Bacillus thuringiensis & 1229 & $N / A$ & Lepidoptera \\
\hline
\end{tabular}

\section{Analysis of Cry proteins structural domains}

Primary sequences of 58 Cry proteins with different specificity in the 3 insect orders were further analyzed to annotate different structural domains involved in various biological processes (Tables 4, 5, and 6). Domain analysis was revealed that most of the Cry proteins have 3 structural domains such as Endotoxin_N (PF03944), Endotoxin_M (PF00555), and Endotoxin_C (PF03945) (Tables 4, 5, and 6). It was also observed that Endotoxin_N, Endotoxin_M, and Endotoxins_C are presented in $\mathrm{N}$ terminus, middle, and $\mathrm{C}$-terminal region of the protein
(Figs. 1 and 2), respectively. Generally, N-terminal helical domain involves in membrane insertion and pore formation whereas middle and $\mathrm{C}$-terminal domains have a vital role in receptor bindings.

\section{Evolutionary analysis of Cry proteins and domains}

Evolutionary tree was constructed for 58 Cry proteins using MEGA $X$ tool after elimination of all residual positions containing gaps and was further analyzed (Fig. 3). The tree is clustered into 3 major groups such as clusters I, II, and III. Cluster I (red color) consists of Cry proteins 
Table 4 List of cry protein domains having toxicity for Coleoptera insect order

\begin{tabular}{|c|c|c|c|c|c|c|c|c|c|c|c|}
\hline \multirow[t]{2}{*}{ Protein name } & \multirow{2}{*}{$\begin{array}{l}\text { Uniprot } \\
\text { accession } \\
\text { No }\end{array}$} & \multirow{2}{*}{$\begin{array}{l}\text { Sequence } \\
\text { length }\end{array}$} & \multicolumn{2}{|c|}{ Endotoxin_N } & \multirow[t]{2}{*}{$E$ value } & \multicolumn{2}{|c|}{ Endotoxin_M } & \multirow[t]{2}{*}{$E$ value } & \multicolumn{2}{|c|}{ Endotoxin_C } & \multirow[t]{2}{*}{$E$ value } \\
\hline & & & Start & End & & Start & End & & Start & End & \\
\hline cry3Ba_coleoptera & P17969 & 659 & 73 & 296 & $4.4 \mathrm{e}-30$ & 304 & 510 & $9.6 \mathrm{e}-62$ & 520 & 659 & $2.5 e-41$ \\
\hline cry3Aa_coleoptera_1 & P0A379 & 644 & 65 & 287 & $3.3 e-30$ & 295 & 499 & $2.1 e-62$ & 509 & 644 & $7.6 e-41$ \\
\hline cry3Aa_coleoptera_2 & P0A381 & 644 & 65 & 287 & $3.3 e-30$ & 295 & 499 & $2.1 e-62$ & 509 & 644 & $7.6 e-41$ \\
\hline cry3Aa_coleoptera_3 & P0A380 & 644 & 65 & 287 & $3.3 e-30$ & 295 & 499 & $2.1 e-62$ & 509 & 644 & $7.6 e-41$ \\
\hline cry3Ca_coleoptera & Q45744 & 649 & 64 & 285 & $2.9 e-32$ & 293 & 502 & $3.6 e-58$ & 512 & 649 & $1 e-39$ \\
\hline cry8Aa_coleoptera & Q45704 & 1157 & 69 & 289 & $1.1 \mathrm{e}-27$ & 297 & 516 & $2.6 e-44$ & 526 & 662 & $6.8 \mathrm{e}-46$ \\
\hline cry8Ba_coleoptera & Q45705 & 1169 & 80 & 290 & $8.8 \mathrm{e}-39$ & 298 & 512 & $7.3 e-54$ & 522 & 658 & $9.3 e-36$ \\
\hline cry7Ab_coleoptera_1 & Q45708 & 1138 & 61 & 278 & $1.8 \mathrm{e}-29$ & 286 & 487 & $9.7 e-59$ & 497 & 637 & $1.5 \mathrm{e}-42$ \\
\hline cry8Ca_coleoptera & Q45706 & 1160 & 77 & 290 & $3.5 e-26$ & 298 & 503 & $5.7 e-50$ & 513 & 659 & $2.6 \mathrm{e}-33$ \\
\hline cry9Da_coleoptera & 006014 & 1169 & 73 & 293 & $5.6 \mathrm{e}-26$ & 301 & 521 & $1.7 e-57$ & 531 & 668 & $2.7 e-48$ \\
\hline cry7Ab_coleoptera_2 & Q45707 & 1138 & 61 & 278 & $2.6 e-30$ & 286 & 487 & $2.5 e-57$ & 497 & 637 & $3.7 e-41$ \\
\hline cry34Ab1_coleoptera ${ }^{a}$ & Q939T0 & 123 & - & - & - & - & - & - & - & - & - \\
\hline cry7Aa_coleoptera & Q03749 & 1138 & 63 & 278 & $1.5 e-27$ & 286 & 487 & $2 e-59$ & 497 & 637 & $1.9 \mathrm{e}-42$ \\
\hline cry35Ab1_coleopterab & Q939S9 & 383 & - & - & - & - & - & - & - & - & - \\
\hline cry3Bb1_coleoptera & Q06117 & 652 & 65 & 288 & $1.2 \mathrm{e}-29$ & 296 & 502 & $1.6 e-63$ & 512 & 651 & $1 e-42$ \\
\hline
\end{tabular}

Toxin_10: domain position (174-348)

${ }^{a}$ Aegerolysin: domain position (3-118)

${ }^{\mathrm{b}} \mathrm{RICIN}$ : domain position (4-138)

having toxicity for insect order Lepidoptera. Interestingly, all Cry1A, Cry1D, Cry1E, Cry1H, Cry1F, Cry1G, Cry1C, and Cry1J are fallen in this cluster. However, in cluster II (green color), all Cry proteins show toxicity towards Coleoptera are joined together along with Cry11D, Cry9Ca, Cry1Be, Cry $1 \mathrm{Ka}, \mathrm{Cry} 1 \mathrm{Bd}, \mathrm{Cry} 1 \mathrm{Bb}$, and Cry9Ea. But, Cry9Aa and Cry2Ab targets for Lepidoptera and Cry8Ca targets Coleoptera were obtained as a member of cluster III (blue color) which shows toxicity towards Diptera. Interestingly, lepidopteran target Cry15Aa and coleopteran targets Cry34Ab1 and Cry35Ab1 were seemed as an out-group in the whole tree and were found to be distantly related from all the Cry protein sequences. The Cry proteins for the specific order were found to be diverged from the own group and placed in different insect orders indicate that they might have insecticidal property for more than one insect order. Besides, in order to understand the insect specificity of these Cry proteins, the functional domain regions were also taken for studying the divergence among them. The phylogenetic tree of the domain Endotoxin_N, Endotoxin_M, and Endotoxin_C was constructed using MEGA X software and presented (Fig. 4).

Table $\mathbf{5}$ List of cry protein domains having toxicity for Diptera insect order

\begin{tabular}{|c|c|c|c|c|c|c|c|c|c|c|c|}
\hline \multirow[t]{2}{*}{ Protein name } & \multirow{2}{*}{$\begin{array}{l}\text { Uniprot } \\
\text { accession } \\
\text { no. }\end{array}$} & \multirow{2}{*}{$\begin{array}{l}\text { Sequence } \\
\text { length }\end{array}$} & \multicolumn{2}{|c|}{ Endotoxin_N } & \multirow[t]{2}{*}{$E$ value } & \multicolumn{2}{|c|}{ Endotoxin_M } & \multirow[t]{2}{*}{$E$ value } & \multicolumn{2}{|c|}{ Endotoxin_C } & \multirow[t]{2}{*}{$E$ value } \\
\hline & & & Start & End & & Start & End & & Start & End & \\
\hline cry11Bb_diptera & Q9ZIU5 & 750 & 56 & 240 & $2.7 e-21$ & - & - & - & - & - & - \\
\hline cry19Aa_diptera & O32307 & 648 & 69 & 292 & $4.3 e-25$ & 300 & 502 & $3.6 e-49$ & 512 & 648 & $1.2 e-31$ \\
\hline cry11Ba_diptera & Q45730 & 724 & 55 & 240 & $1.2 \mathrm{e}-18$ & - & - & - & - & - & - \\
\hline cry4Ba_diptera & P05519 & 1136 & 52 & 268 & $1.1 \mathrm{e}-26$ & 283 & 470 & $2.1 e-40$ & 480 & 634 & $2.2 \mathrm{e}-40$ \\
\hline cry10Aa_diptera & P09662 & 675 & 95 & 300 & $2.9 \mathrm{e}-23$ & 308 & 500 & $5.9 e-37$ & 510 & 650 & $2.8 \mathrm{e}-35$ \\
\hline cry4Aa_diptera & P16480 & 1180 & 80 & 314 & $1.3 e-21$ & 322 & 528 & $2.6 e-54$ & 538 & 678 & $2.6 e-40$ \\
\hline cry11Aa_diptera & P21256 & 643 & 41 & 240 & $5.1 e-23$ & - & - & - & - & - & - \\
\hline cry27Aa_diptera & Q9S597 & 826 & 92 & 302 & $6.4 \mathrm{e}-23$ & 311 & 499 & $5.1 e-19$ & 524 & 683 & $8.5 e-34$ \\
\hline cry19Ba_diptera & 086170 & 682 & 71 & 280 & $3.2 \mathrm{e}-32$ & 293 & 496 & $2.2 \mathrm{e}-48$ & 506 & 638 & $9.1 e-34$ \\
\hline cry20Aa_diptera & 032321 & 753 & 67 & 280 & $1.7 e-22$ & 288 & 485 & $3.2 e-44$ & 495 & 631 & $1.5 e-26$ \\
\hline
\end{tabular}


Table 6 List of protein domains having toxicity for Lepidoptera insect order

\begin{tabular}{|c|c|c|c|c|c|c|c|c|c|c|c|}
\hline \multirow[t]{2}{*}{ Protein name } & \multirow{2}{*}{$\begin{array}{l}\text { Uniprot } \\
\text { accession } \\
\text { no. }\end{array}$} & \multirow{2}{*}{$\begin{array}{l}\text { Sequence } \\
\text { length }\end{array}$} & \multicolumn{2}{|c|}{ Endotoxin_N } & \multirow[t]{2}{*}{$E$ value } & \multicolumn{2}{|c|}{ Endotoxin_M } & \multirow[t]{2}{*}{$E$ value } & \multicolumn{2}{|c|}{ Endotoxin_C } & \multirow[t]{2}{*}{$E$ value } \\
\hline & & & Start & End & & Start & End & & Start & End & \\
\hline cry1Bd_lepidoptera & Q9ZAZ5 & 1231 & 59 & 275 & $2.4 \mathrm{e}-26$ & 283 & 495 & $2.1 \mathrm{e}-65$ & 505 & 643 & $1.1 \mathrm{e}-38$ \\
\hline cry1Gb_lepidoptera & Q9ZAZ6 & 1169 & 45 & 245 & $4.7 e-22$ & 253 & 449 & $4.8 e-54$ & 459 & 596 & $3.7 e-48$ \\
\hline cry9Ca_lepidoptera & Q45733 & 1157 & 70 & 290 & $1.6 e-29$ & 298 & 505 & $3.8 \mathrm{e}-56$ & 515 & 658 & $6 e-41$ \\
\hline cry15Aa_lepidoptera ${ }^{a}$ & Q45729 & 340 & - & - & - & - & - & - & - & - & - \\
\hline cry1Aa_lepidoptera_1 & P0A369 & 934 & 46 & 251 & $1 e-31$ & 259 & 460 & $4.9 e-53$ & 470 & 607 & $8.9 e-47$ \\
\hline cry1Ka_lepidoptera & Q45715 & 1215 & 56 & 276 & $2.7 e-25$ & 284 & 490 & $6.1 e-60$ & 500 & 637 & $4.8 e-40$ \\
\hline cry1Hb_lepidoptera & Q45718 & 1155 & 48 & 248 & $3.6 e-28$ & 256 & 454 & $2.3 e-58$ & 464 & 596 & $3.2 e-38$ \\
\hline cry1Fb_lepidoptera & O66377 & 1169 & 44 & 249 & $5.2 \mathrm{e}-33$ & 257 & 454 & $6.2 e-54$ & 464 & 600 & 1.6e-49 \\
\hline cry1Ab_lepidoptera & P0A370 & 1155 & 46 & 251 & $8.2 \mathrm{e}-31$ & 259 & 461 & $1.6 e-57$ & 471 & 608 & $2.6 e-46$ \\
\hline cry1Aa_lepidoptera_2 & P0A366 & 1176 & 46 & 251 & $2.3 e-31$ & 259 & 460 & $1 e-52$ & 470 & 607 & $2.6 e-46$ \\
\hline cry2Ab_lepidoptera & P21254 & 633 & 53 & 263 & $2.5 e-31$ & - & - & - & - & - & - \\
\hline cry1Ac_lepidoptera & P05068 & 1178 & 46 & 251 & $3.5 e-31$ & 259 & 461 & $3.6 e-58$ & 471 & 609 & $1.9 e-36$ \\
\hline cry1Ea_lepidoptera & Q57458 & 1171 & 44 & 250 & $3 e-28$ & 258 & 454 & $1.6 e-60$ & 464 & 601 & $2.2 e-39$ \\
\hline cry9Aa_lepidoptera & Q99031 & 1156 & 61 & 287 & $5.7 e-17$ & 295 & 510 & $5.3 e-46$ & 520 & 656 & $1.4 \mathrm{e}-36$ \\
\hline cry1Aa_lepidoptera_3 & P0A368 & 1176 & 46 & 251 & $2.3 e-31$ & 259 & 460 & $1 e-52$ & 470 & 607 & $2.6 e-46$ \\
\hline cry1Ca_lepidoptera_1 & P0A375 & 1189 & 42 & 250 & $9.1 \mathrm{e}-28$ & 260 & 457 & $1.4 \mathrm{e}-51$ & 467 & 616 & $1.5 e-37$ \\
\hline cry1Ae_lepidoptera & Q03748 & 1181 & 46 & 251 & $5.5 e-29$ & 259 & 461 & $3.7 e-57$ & 471 & 608 & $2.8 e-46$ \\
\hline cry1Ca_lepidoptera_2 & P0A376 & 1189 & 42 & 250 & $6.2 \mathrm{e}-28$ & 260 & 457 & $5.1 e-54$ & 467 & 616 & $1.5 e-37$ \\
\hline cry1Aa_lepidoptera_4 & P0A367 & 1176 & 46 & 251 & $3.4 \mathrm{e}-31$ & 259 & 460 & $1 e-52$ & 470 & 607 & $2.6 e-46$ \\
\hline cry1Ad_lepidoptera & Q03744 & 1179 & 46 & 251 & $9.6 e-31$ & 259 & 460 & $5.8 e-53$ & 470 & 607 & $1.2 \mathrm{e}-44$ \\
\hline cry1Da_lepidoptera & P19415 & 1165 & 45 & 250 & $1.9 e-32$ & 258 & 450 & $1.4 \mathrm{e}-54$ & 460 & 592 & $7 e-39$ \\
\hline cry1Eb_lepidoptera & Q03745 & 1174 & 42 & 249 & $8.5 e-31$ & 257 & 453 & $2 e-59$ & 463 & 599 & $1.4 \mathrm{e}-39$ \\
\hline cry1Fa_lepidoptera & Q03746 & 1174 & 44 & 249 & $8.7 e-33$ & 257 & 454 & $1.4 e-53$ & 464 & 601 & $2.2 e-43$ \\
\hline cry9Ea_lepidoptera & Q9ZNL9 & 1150 & 71 & 293 & $3.9 e-22$ & 301 & 505 & $2.6 e-47$ & 515 & 651 & 2.1e-42 \\
\hline cry1Ga_lepidoptera & Q45746 & 1166 & 46 & 245 & $1.7 e-26$ & 253 & 446 & $9.4 e-51$ & 456 & 593 & $2.9 e-49$ \\
\hline cry1ld_lepidoptera & Q9XDL1 & 719 & 64 & 279 & $1.8 \mathrm{e}-33$ & 287 & 497 & $6 e-65$ & 507 & 644 & $2.3 e-48$ \\
\hline cry1Be_lepidoptera & O85805 & 1227 & 60 & 275 & $5.8 e-25$ & 283 & 493 & $6.9 e-65$ & 503 & 639 & $5.8 e-39$ \\
\hline cry1Ha_lepidoptera & Q45748 & 1172 & 48 & 249 & $8.2 \mathrm{e}-28$ & 257 & 455 & $8.3 e-58$ & 465 & 599 & $5.6 e-35$ \\
\hline cry1Jb_lepidoptera & Q45716 & 1170 & 46 & 250 & $6.9 e-27$ & 258 & 449 & $8.6 e-57$ & 459 & 596 & $1.1 e-43$ \\
\hline cry1Ag_lepidoptera & Q9S515 & 1176 & 46 & 251 & $2.9 e-31$ & 259 & 454 & $2 e-44$ & 473 & 607 & $1.6 e-37$ \\
\hline cry1Db_lepidoptera & Q45747 & 1160 & 45 & 250 & $4 e-31$ & 258 & 450 & $4 e-54$ & 460 & 592 & $1.7 e-38$ \\
\hline cry1Ja_lepidoptera & Q45738 & 1167 & 46 & 250 & $6.8 \mathrm{e}-27$ & 258 & 449 & $1.7 e-54$ & 459 & 595 & $8.1 e-49$ \\
\hline cry1Bb_lepidoptera & Q45739 & 1229 & 60 & 275 & $2.4 \mathrm{e}-26$ & 283 & 495 & $2.1 e-65$ & 505 & 641 & $6.3 e-46$ \\
\hline
\end{tabular}

aETX_MTX2: domain position (40-265)

The Cry protein sequence Endotoxin_N domains (55 numbers) was clustered into 3 major groups. The evolutionary analysis (Fig. 4a) depicted that the divergence pattern of all the domain sequences as similar with that of all Cry protein sequences and presented in (Fig. 3). Evolutionary analysis of Endotoxin_M domains (51 numbers) present in Crystal protein target for all the 3 insect orders depicted in (Fig. 4b) showed a similar type of divergence in the phylogenetic tree as that of two predicted tree for Endotoxin_N and total Cry protein sequence. Phylogenetic tree involving Endotoxin_C domains (51 numbers) was revealed that all Cry1 and Cry9 targets for lepidopterans are grouped into a single cluster, except for Cry1C, Cry1B, Cry1E, and Cry1Ac, which are found to be clustered into the second group of Cry protein, which targets the coleopterans. However, all Cry proteins for dipteral were clustered into the same group, except for Cry8Ba, which targets the coleopteran (Fig. 4c). 

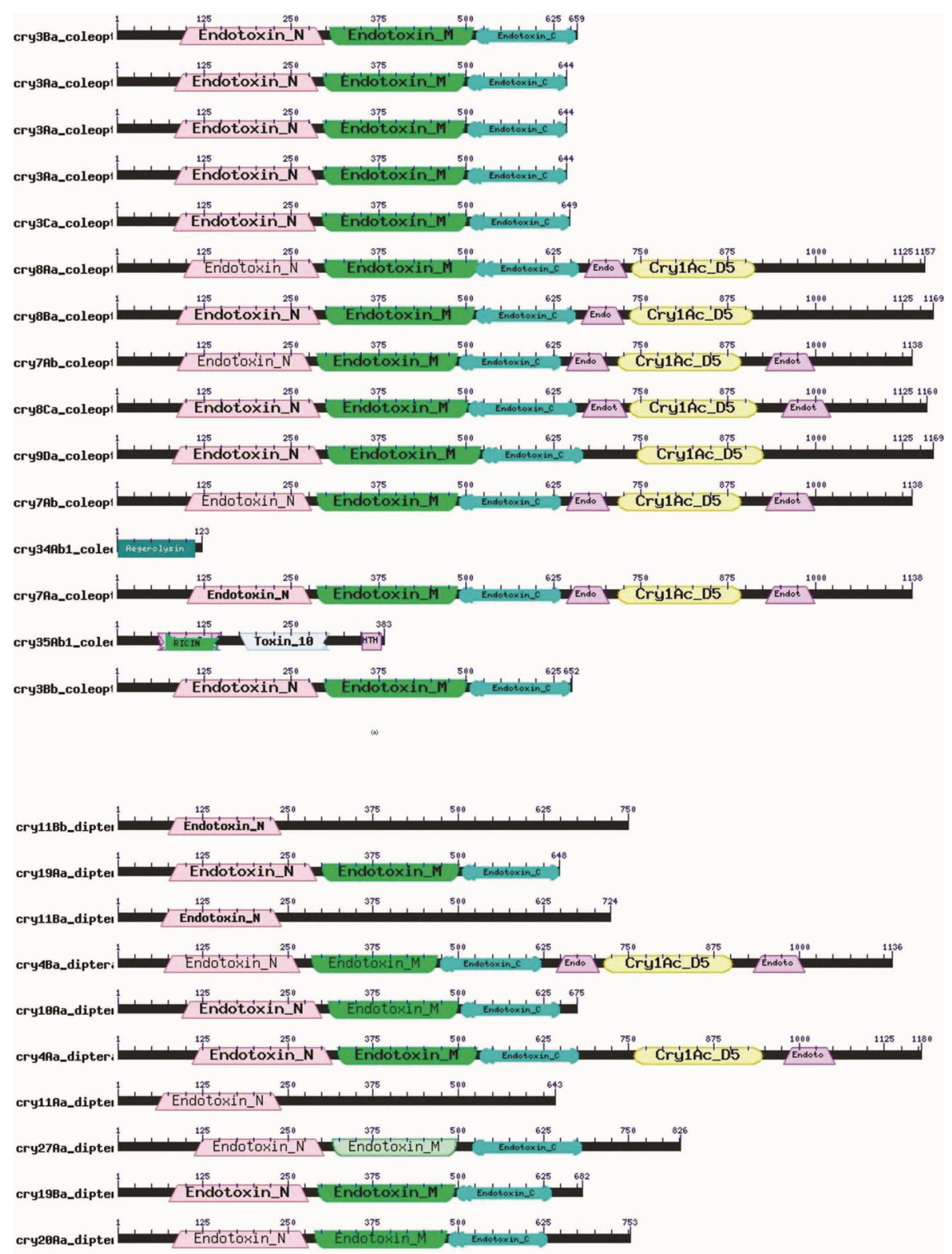

Fig. 1 a NCBI batch conserved domain-search result for Coleoptera insect order. b NCBI batch conserved domain-search for Diptera insect order

\section{Analysis of Cry protein structure}

Structural analysis of Cry protein sequences was performed to understand its function in a better way. It has been observed that most of the Cry proteins do not have experimentally solved 3-dimensional structures in the RCSB PDB, except for Cry3Aa, Cry34Ab1, Cry35Ab1, and Cry3Bb1 for Coleoptera (Fig. 5a), Cry4Ba and Cry4Aa for Diptera (Fig. 5b), and Cry1Aa, Cry1Ac, Cry1Da, Cry1Fa, and Cry1Be for Lepidoptera (Fig. 6a,b). The rest 47 unsolved 3-dimensional structures of Cry proteins belongs to 3 different insect orders predicted through Phyre2 homology modeling server, i.e., 11 numbers of model structure for coleopteran, 08 numbers of model structures for dipterans, and 28 numbers of model structure for lepidopterans. Structural analysis of Cry proteins for coleopteran's group revealed that the proteins having Endotoxin_N, Endotoxin_M, and Endotoxin_C at sequence level corresponding to domain I, domain II, and domain III, respectively, at their structural levels. Domain I regions found to be observed starting from 60 to 300 amino acids at the $\mathrm{N}$-terminal region of the Cry proteins and consists of $\alpha$-helices $(\sim 8$ 


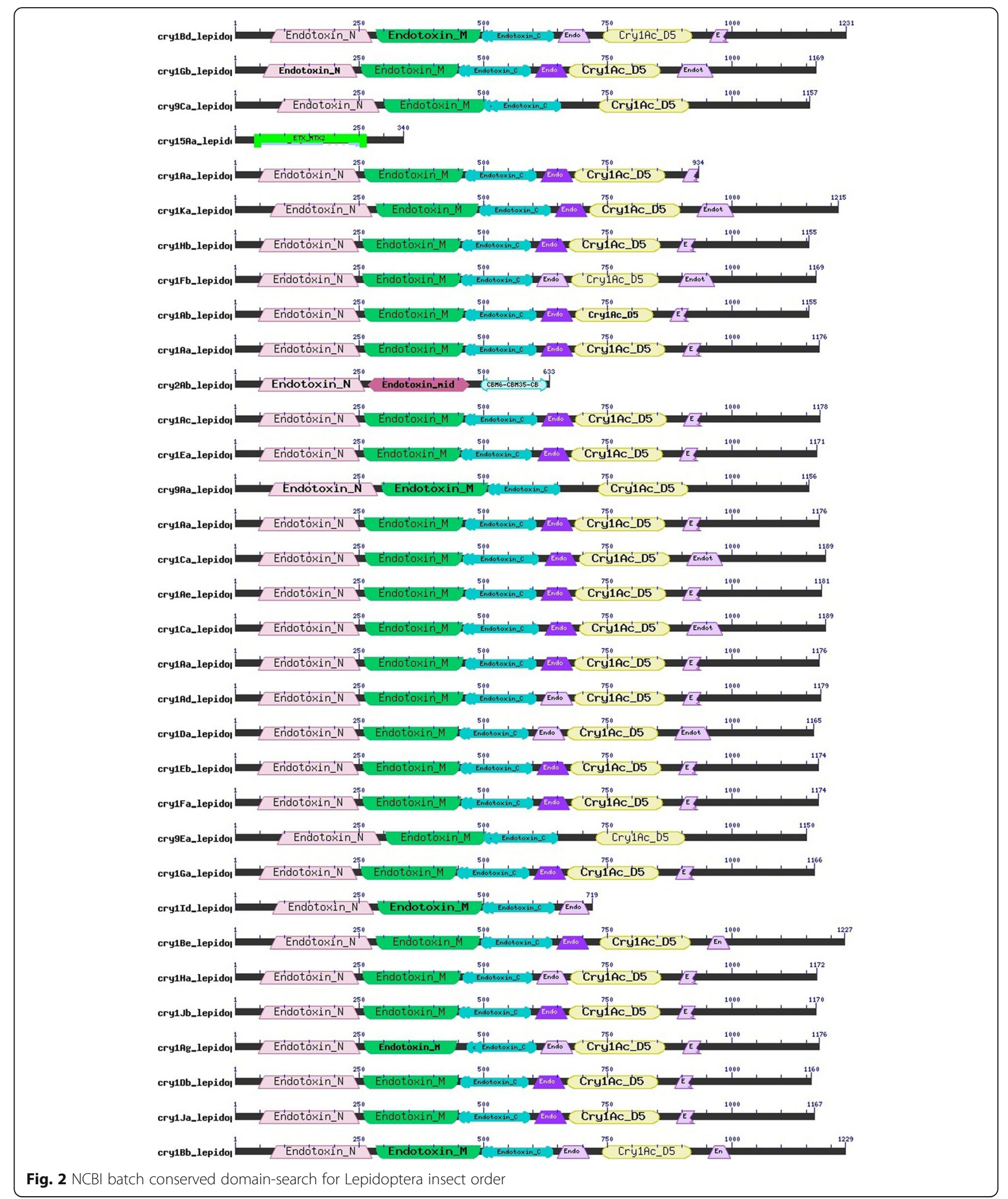

numbers) whereas amino acid position 280-510 form domain II which consists of ant parallel $\beta$-sheets and short helices. Domain III is a $\beta$-sandwich of two ant parallel highly twisted $\beta$-sheets and comprises 490-670 amino acid residues, corresponding to Endotoxin_C domain of the Cry protein at the C-terminal region. By doing structure-structure alignment involving both experimental and structural Cry proteins of Coleopteran 


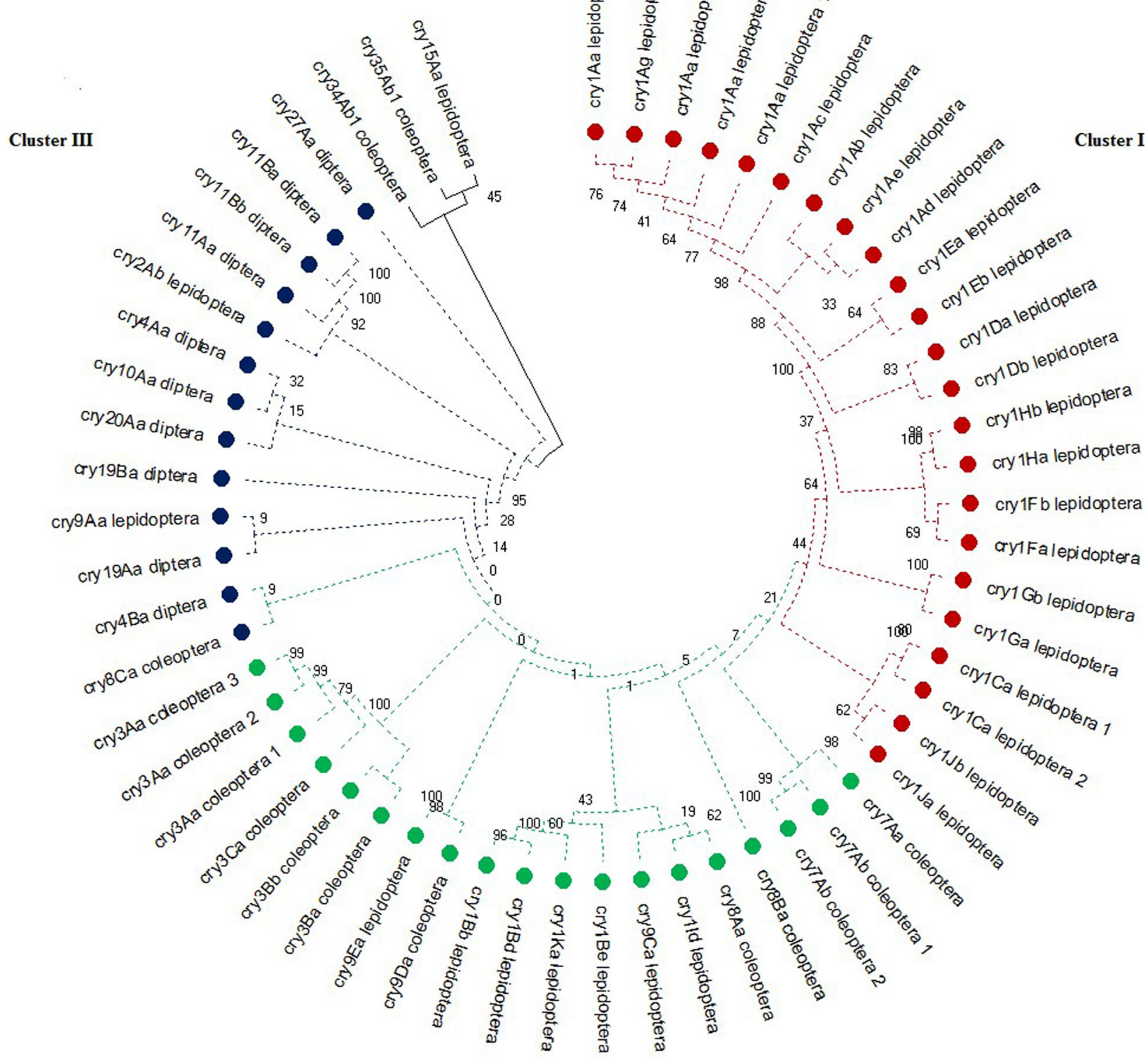

Cluster II

Fig. 3 Phylogenetic tree using MEGA X showing 58 numbers of cry proteins having toxicity for three insect orders

group depicted that all 15 three-dimensional structures for Coleopteran are clustered into two major groups comprising Cry7Aa, Cry9Da, Cry8Ba, Cry8Ca, Cry7Ab ( 2 numbers), and Cry8Aa in one group whereas Cry3Aa (3 numbers), Cry3Ca, Cry3Ba, and Cry3 in another group whereas Cry34Ab1 and Cry35Ab1 are observed as out-groups (Fig. 7a). The divergence level may be due to the absence of Endotoxin_N, Endotoxin_M, and Endotoxin_C domains. In case of Cry proteins having specificity for Diptera, 8 model structures were predicted such as Cry10Aa, Cry11Aa, Cry11Ba, Cry11Bb, Cry19Aa,
Cry19Ba, Cry20Aa, and Cry27Aa. Three-dimensional structural analysis of both experimental (2 numbers) and model (8 numbers) structure revealed that domain I is present in all the protein which extends from residue $40-302$ is totally $\alpha$-helical and contains around 8 numbers of $\alpha$-helices whereas domain II and domain III span over residue 280-530 and 480-685, respectively, except Cry11Ba and Cry11Aa whereas Endotoxin_M and Endotoxin_C domains are absent. In structural neighbor analysis, all are grouped in single group except Cry4Ba which is found to be an out-group for this group (Fig. 

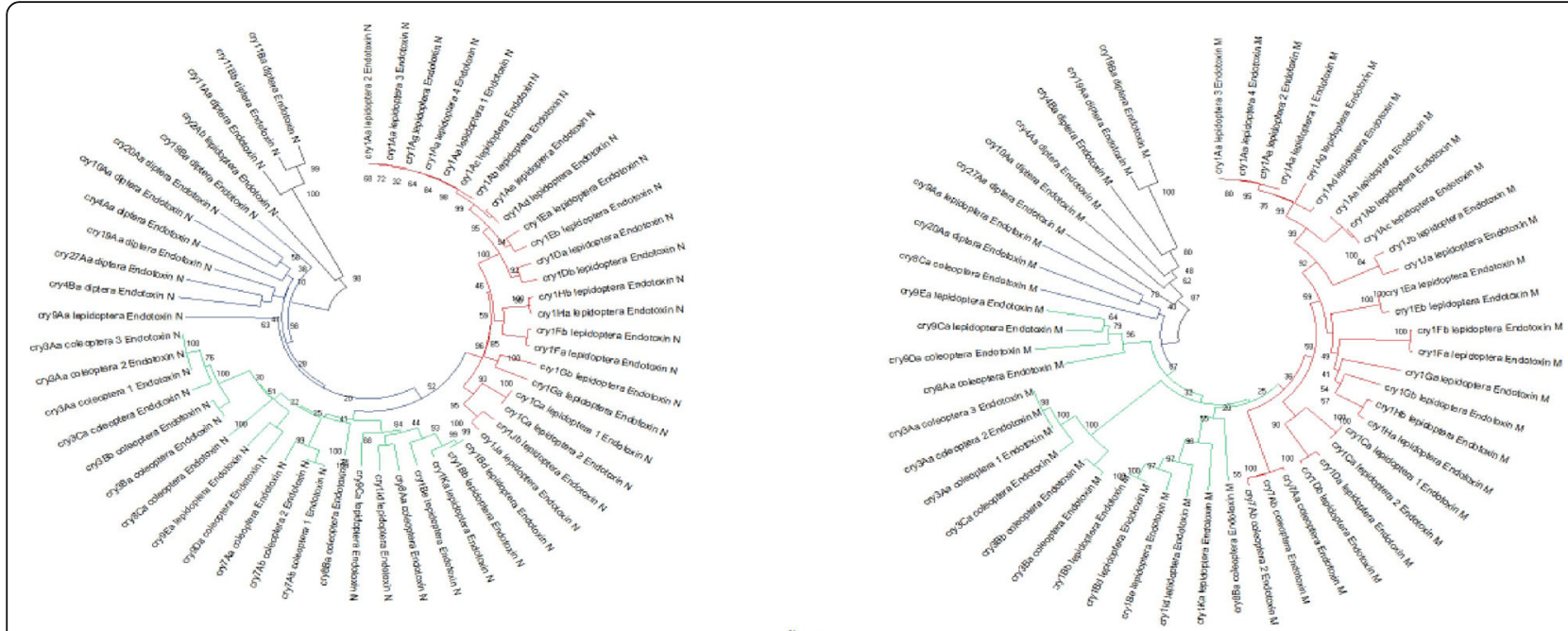

(a)

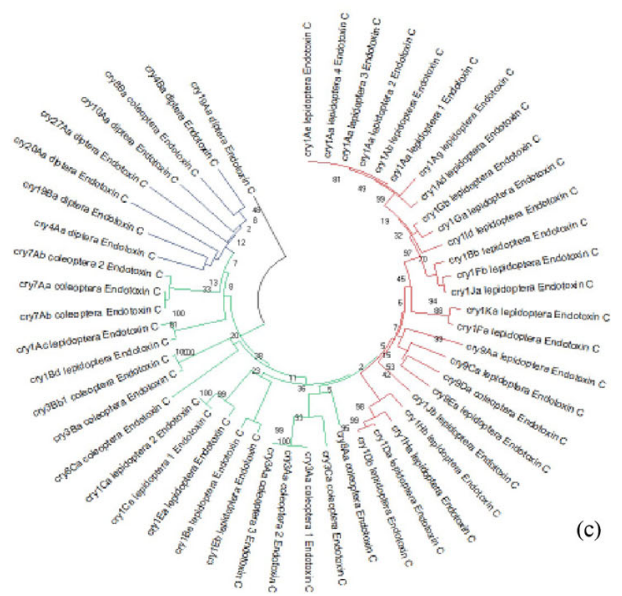

(b)

Fig. 4 a Phylogenetic tree of Cry protein domains for Coleoptera using MEGA X. b Phylogenetic tree of Cry protein domains for Diptera using MEGA X. c Phylogenetic tree of Cry protein domains for Lepidoptera using MEGA X

7b). A total of 28 numbers of predicted protein structures having specificity for lepidopteran's group were predicted. It was revealed that Cry1Aa, Cry1Be, Cry1Ba, and Crylfa have the same type of folding pattern whereas totally different orientation was observed in case of Cry15Aa due to absence of Endotoxin_N, Endotoxin_ $\mathrm{M}$, and Endotoxin_C domains. Different domain regions such as domain I, domain II, and domain III extend from 40 to $295,253-510$, and 458-660, respectively, for lepidopteran's group (Table 6). Alignment of all 28 proteins structures was clustered into one major group leaving behind $\mathrm{Cry} 2 \mathrm{Ab}$ as distantly related to them whereas Cry15Aa was observed as out-group (Fig. 7c).

$B$. thuringiensis $(B t)$ strains produce a wide variety of proteins having toxicity against diverse insect orders. These toxins classified into 2 major groups: crystal (Cry) and cytolytic (Cyt). More than 700 Cry gene sequences that code for crystal protein (Cry) have been identified in plasmids by several researchers (Höfte and Whiteley 1989; Schnepf et al. 1998; Van Frankenhuyzen 2009).
Many Cry proteins are reported to have useful insecticidal properties for controlling insect pests in agriculture (Sanchis and Bourguet 2008). However, strong cytocidal activities have also been noticed against vertebrates (Palma et al. 2014). Primary protein sequence database search revealed that there are 58 numbers of Cry proteins showing specificity towards the 3 major insect orders: Coleoptera, Diptera, and Lepidoptera (Donovan et al. 2016; Sanchis and Bourguet 2008; Naimov et al. 2008). Sequences of different insect orders showed different sequence's length. All Cry1, Cry2, and Cry15 groups showed toxicity towards lepidopteran insects, whereas Cry9 group showed toxicity towards Lepidoptera and Coleoptera. Similarly, Cry proteins such as Cry3, Cry7, Cry8, Cry34, and Cry35 groups were found to have specificity for the coleopterans. Cry4, Cry10, Cry11, Cry19, Cry20, and Cry27 groups exhibit insecticidal activity against the insect belonging to dipteran's order. The classification of Cry proteins and their insecticidal activity against specific insect orders have been 

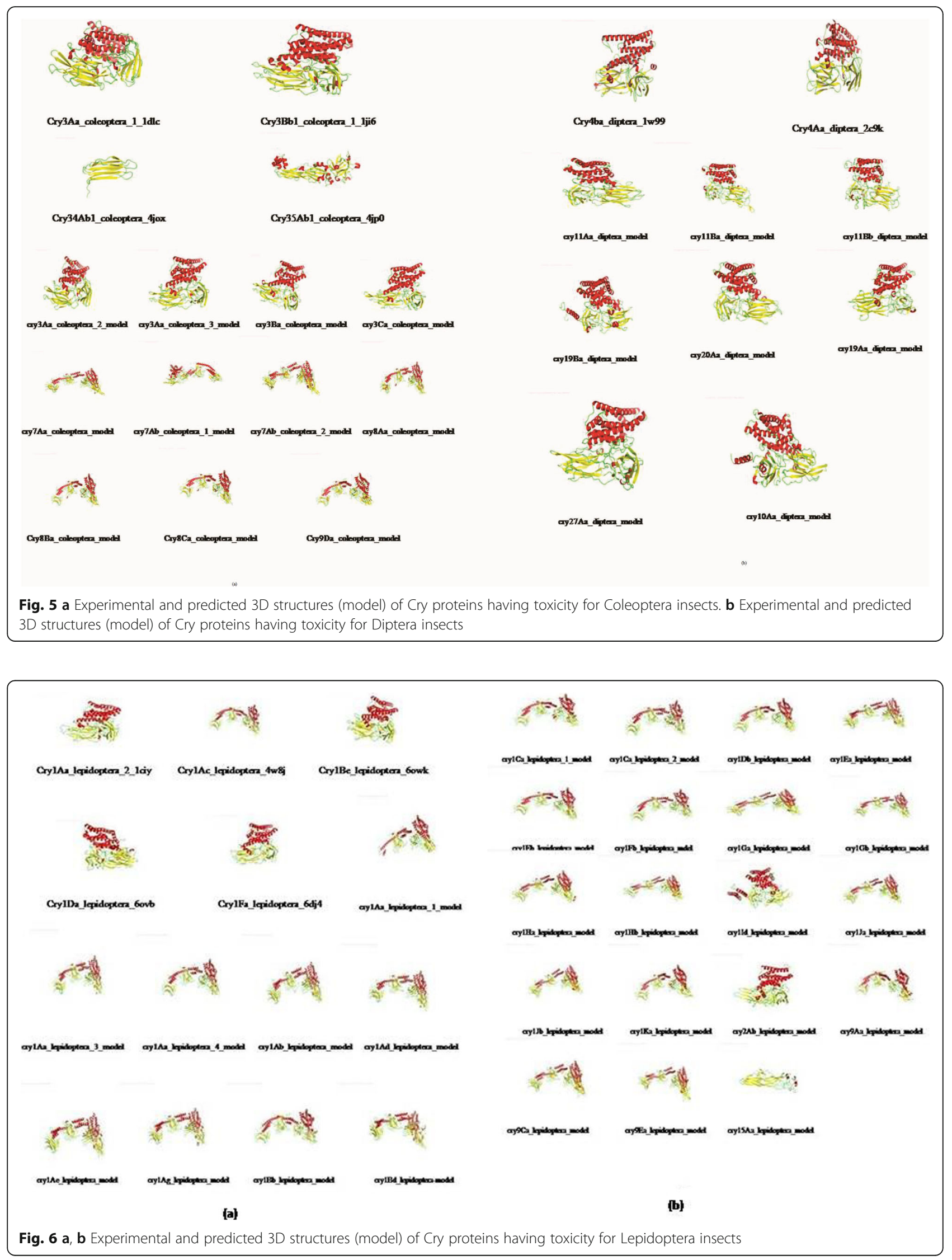


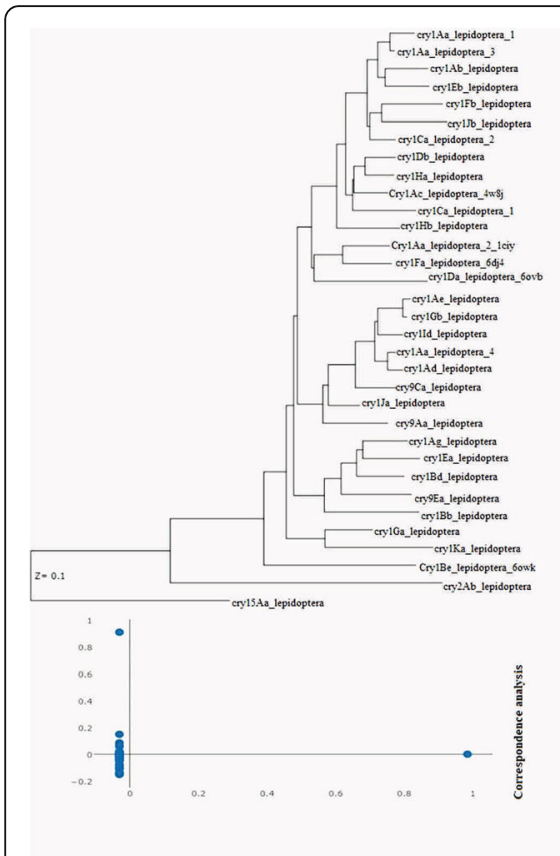

(a)

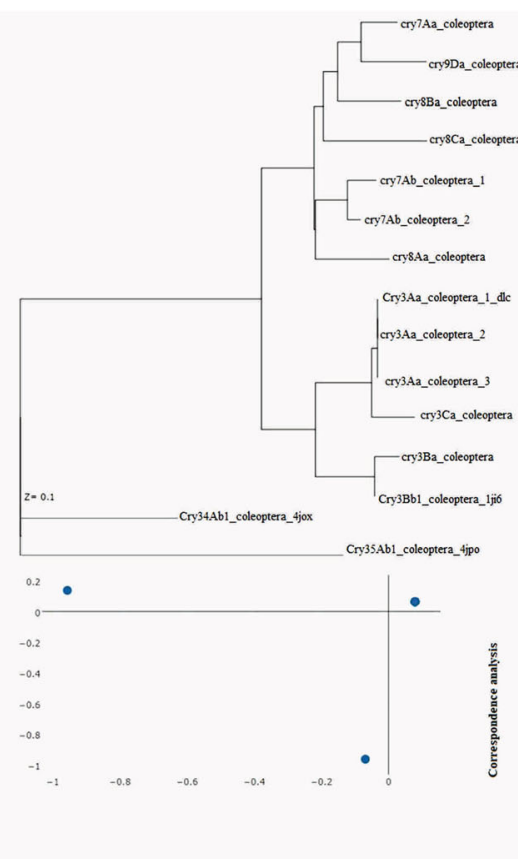

(b)

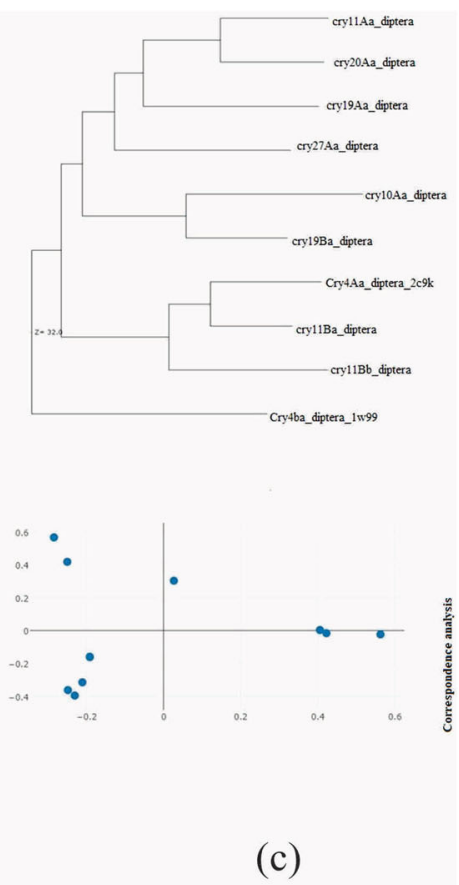

(c)

Fig. 7 a 3D Structure alignment result using Phyre ${ }^{2}$ server of Cry proteins (Coleoptera). b 3D Structure alignment result using Phyre ${ }^{2}$ server of Cry Proteins (Diptera). c 3D Structure alignment result using Phyre ${ }^{2}$ server of Cry Proteins (Lepidoptera)

studied by several researchers (Crickmore 2000; de Maagd et al. 2001). Analysis of evolutionary relationship revealed that Cry proteins such as Cry1Id, Cry9Ca, Cry1Be, Cry1Ka, Cry1Bd, Cry1Bb, and Cry9Ea showed toxicity towards lepidopteran's insects also clustered together with the Cry proteins having toxicity for the coleopterans. This finding is supported by (Crickmore 2000). It has been also suggested that these proteins may have toxicity against both insect orders and was later showed for Cry1B toxin (López-Pazos et al. 2009). Similarly, Cry8Ca proteins of coleopteran's group and Cry2Ab and Cry9Aa for lepidopterans are found to be clustered in Diptera insect group. This phylogenetic relationship of whole Cry proteins could not able to reveal how Cry toxin involves in insect specificity. To validate further, phylogenetic analysis of the 3 structural domains such as domain I (Endotoxin_N), domain II (Endotoxin_M), and domain III (Endotoxin_C) were carried out independently. Divergence pattern were observed similar in case of both sequence level and structural level with minor fluctuation.

As per literature, the domain swapping of different Cry toxin is likely to be an active evolutionary process for determining insect specificity (de Maagd et al. 2001). Threedimensional X-ray crystallography structures of several Cry toxins of $B t$ have been reported in this connection. A total of 11 numbers of three-dimensional structures of the Cry proteins were retrieved from RCSB PDB out of which
4 proteins for Coleoptera, 2 for Diptera, and 5 for Lepidoptera. Homology model structure for 47 Cry proteins were predicted using web based Phyre 2 tool to study the structural arrangements of 3 different domains. All experimental and predicted models of Cry proteins revealed that the domain $\mathrm{I}$ is present in $\mathrm{N}$-terminal region having $\alpha$ helices, domain II consists of three antiparallel $\beta$-sheets, and domain III consists of two twisted anti-parallel $\beta$ sheets forming a sandwich. This observations and findings are also supported by different published reports (Grochulski et al. 1995; de Maagd et al. 2001, and Gouet et al. 2003). Structure-structure alignment was also carried out involving all the 58 numbers of Cry proteins (experimental and model 3-dimensional structures) in order to understand the divergence among each other. In case of Coleoptera insect order, 3-dimensional structures of Cry34Ab1 and Cry35Ab1 were observed as out-group. In case of Diptera, 3-dimensional structures of Cry4Ba were found diverged from other Cry proteins whereas Cry2Ab protein of Lepidoptera group noticed to be diverged from other group of proteins. However, Cry15aa was predicted as an out-group in compare to other Cry proteins.

\section{Conclusions}

Bacillus thuringiensis (Bt) synthesizes various insecticidal proteins and thus recommended as potential bio control agent against various insect pests in agriculture. The evolution and diversification of these Cry proteins have 
been studied extensively by various researchers to discover the existence of important determinants, which confer insect specificity for improvement of its insecticidal activity. There are total 58 numbers of different Cry protein groups belong to major three insect orders: Coleoptera, Diptera, and Lepidoptera were retrieved and analyzed both at structural and sequence level. Structural and functional analysis was performed to understand the domain arrangements at sequence and structural level involving both experimental and predicted 3D models. Cry proteins having toxicity for a specific insect order are grouped accordingly. Threedimensional structure analysis of both experimental and predicted models revealed that the Cry proteins might have toxicity for a specific insect order differ in their structural arrangements and is observed in 3 different groups. It could be hypothesized that an inner-molecular domain shift or domain insertion/deletion might have taken place during the evolutionary process, which consequently causes structural and functional divergence of $B t$. These findings lead to understand the wide diversity of insecticidal proteins and their application as biopesticides in agriculture.

\section{Abbreviations}

Bt: Bacillus thuringiensis; PFTs: Pore-forming toxins; 3D: Three-dimensional; CADs: Cadherin-like proteins; APN: Aminopeptidase N; UniProt: Universal Protein Resource; NCBI : National Center for Biotechnology Information; CDSearch: Conserved Domain-Search; RCSB-PDB: Research Collaboratory for Structural Bioinformatics-Protein Data Bank; MEGA X: Molecular Evolutionary Genetics Analysis X; Phyre 2 : Protein Homology/analogY Recognition Engine V 2.0

\section{Acknowledgements}

Financial assistance to the Center of Excellence in Environment and Public Health by the Higher Education Department, Government of Odisha under OHEPEE is grateful acknowledged (HE-PTC-WB-02017).

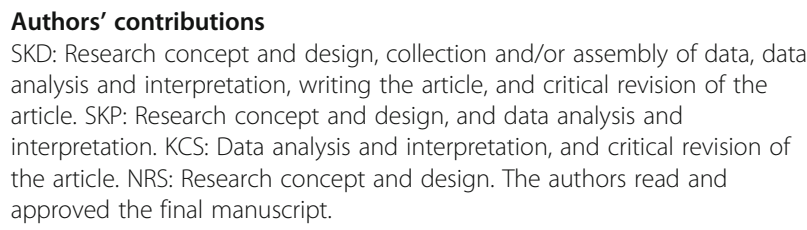
analysis and interpretation, writing the article, and critical revision of the article. SKP: Research concept and design, and data analysis and interpretation. KCS: Data analysis and interpretation, and critical revision of the article. NRS: Research concept and design. The authors read and approved the final manuscript.

\section{Funding}

Not applicable.

\section{Availability of data and materials}

The datasets used and/or analyzed during the current study are available in the UniProtKB (The Universal Protein Resource Knowledgebase).

\section{Ethics approval and consent to participate}

Not applicable.

\section{Consent for publication}

Not applicable.

\section{Competing interests}

The authors declare that they have no competing interests.

\section{Author details}

'Department of Bioinformatics, Centre for Post Graduate Studies, Odisha University of Agriculture \& Technology, Bhubaneswar, Odisha 751003, India. ${ }^{2}$ School of Life Sciences, Ravenshaw University, Cuttack, Odisha 753003, India. ${ }^{3}$ Department of Agricultural Biotechnology, College of Agriculture, Odisha University of Agriculture \& Technology, Bhubaneswar, Odisha 751003, India.

${ }^{4}$ Department of Botany and Center of Excellence in Environment and Public Health, Ravenshaw University, Cuttack, Odisha 753003, India.

Received: 27 August 2020 Accepted: 17 February 2021

Published online: 01 March 2021

\section{References}

Bayyareddy K, Andacht TM, Abdullah MA, Adang MJ (2009) Proteomic identification of Bacillus thuringiensis subsp. israelensis toxin Cry4Ba binding proteins in midgut membranes from Aedes aegyptiLinnaeus (Diptera, Culicidae) larvae. Insect Biochem Mol Biol 39(4):279-286. https://doi.org/10.1 016/j.ibmb.2009.01.002

Boonserm P, Davis P, Ellar DJ, Li J (2005) Crystal structure of the mosquitolarvicidal toxin Cry4Ba and its biological implications. J Mol Biol 348(2):363382. https://doi.org/10.1016/j.jmb.2005.02.013

Boonserm P, Mo M, Angsuthanasombat C, Lescar J (2006) Structure of the functional form of the mosquito larvicidal Cry4Aa toxin from Bacillus thuringiensis at a 2.8-angstrom resolution. J Bacteriol 188(9):3391-3401. https://doi.org/10.1128/JB.188.9.3391-3401.2006

Bravo A, Gill SS, Soberón M (2007) Mode of action of Bacillus thuringiensis Cry and Cyt toxins and their potential for insect control. Toxicon 49(4):423-435. https://doi.org/10.1016/j.toxicon.2006.11.022

Bravo A, Gómez I, Porta H, García-Gómez Bl, Rodrigue-Almazan C, Pardo L, Soberón M (2013) Evolution of Bacillus thuringiensis Cry toxins insecticidal activity. J Microbial Biotechnol 6(1):17-26. https://doi.org/10.1111/j.1751-791 5.2012.00342.x

Bravo A, Likitvivatanavong S, Gill SS, Soberón M (2011) Bacillus thuringiensis: a story of a successful bioinsecticide. Insect Biochem Mol Biol 41(7):423-431. https://doi.org/10.1016/j.ibmb.2011.02.006

Choma CT, Kaplan H (1990) Folding and unfolding of the protoxin from Bacillus thuringiensis: evidence that the toxic moiety is present in an active conformation. Biochemistry 29(49):10971-10977. https://doi.org/10.1021/ bi00501a015

Crickmore N (2000) The diversity of Bacillus thuringiensis $\delta$-endotoxins. In: Entomopathogenic bacteria: from laboratory to field application. Springer, Dordrecht, pp 65-79

Crickmore N, Zeigler DR, Feitelson J, Schnepf ES, Van Rie J, Lereclus D, Baum J, Dean DH (1998) Revision of the nomenclature for the Bacillus thuringiensis pesticidal crystal proteins. Microbiol Mol Biol Rev 62(3):807-813. https://doi. org/10.1128/MMBR.62.3.807-813.1998

Crickmore N, Zeigler DR, Schnepf E, Van Rie J, Lereclus D, Baum J (2011) Bacillusthuringiensis toxin. [WWWdocument].URL http://www.lifesci.sussex.ac. uk/Home/Neil_Crickmore/Bt/.

de Maagd RA, Bravo A, Crickmore N (2001) How Bacillus thuringiensis has evolved specific toxins to colonize the insect world. Trends Genet 17(4):193-199. https://doi.org/10.1016/S0168-9525(01)02237-5

Derbyshire DJ, Ellar DJ, Li J (2001) Crystallization of the Bacillus thuringiensis toxin Cry1 Ac and its complex with the receptor ligand $\mathrm{N}$-acetyl-D-galactosamine. Acta Crystallogr D Biol Crystallogr 57(12):1938-1944. https://doi.org/10.1107/ S090744490101040X

Donovan WP, Engleman JT, Donovan JC, Baum JA, Bunkers GJ, Chi DJ, Clinton WP, English L, Heck GR, llagan OM, Krasomil-Osterfeld KC (2016) Discovery and characterization of Sip1A: a novel secreted protein from Bacillus thuringiensis with activity against coleopteran larvae. Appl Microbiol Biotechnol 72(4):713-719. https://doi.org/10.1007/s00253-006-0332-7

Evdokimov AG, Moshiri F, Sturman EJ, Rydel TJ, Zheng M, Seale JW, Franklin S (2014) Structure of the full length insecticidal protein Cry1 A c reveals intriguing details of toxin packaging into in vivo formed crystals. Protein Sci 23(11):1491-1497. https://doi.org/10.1002/pro.2536

Galitsky N, Cody V, Wojtczak A, Ghosh D, Luft JR, Pangborn W, English L (2001) Structure of the insecticidal bacterial $\delta$-endotoxin Cry3Bb1 of Bacillus thuringiensis. Acta Crystallogr D Biol Crystallogr 57(8):1101-1109. https://doi. org/10.1107/S09074444901008186 
Gouet P, Robert X, Courcelle E (2003) ESPript/ENDscript: extracting and rendering sequence and 3D information from atomic structures of proteins. Nucleic Acids Res 31(13):3320-3323. https://doi.org/10.1093/nar/gkg556

Griffitts JS, Haslam SM, Yang T, Garczynski SF, Mulloy B, Morris H, Cremer PS, Dell A, Adang MJ, Aroian RV (2005) Glycolipids as receptors for Bacillus thuringiensis crystal toxin. Science 307(5711):922-925. https://doi.org/10.1126/ science. 1104444

Grochulski P, Masson L, Borisova S, Pusztai-Carey M, Schwartz JL, Brousseau R, Cygler M (1995) Bacillus thuringiensis CrylA (a) insecticidal toxin: crystal structure and channel formation. J Mol Biol 254(3):447-464. https://doi.org/1 0.1006/jmbi.1995.0630

Guo S, Ye S, Liu Y, Wei L, Xue J, Wu H, Song F, Zhang J, Wu X, Huang D, Rao Z (2009) Crystal structure of Bacillus thuringiensis Cry8Ea1: an insecticidal toxin toxic to underground pests, the larvae of Holotrichia parallela. J Struct Biol 168(2):259-266. https://doi.org/10.1016/j.jsb.2009.07.004

Höfte H, Whiteley HR (1989) Insecticidal crystal proteins of Bacillus thuringiensis. Microbiol Mol Biol Rev 53(2):242-255

Hui F, Scheib U, Hu Y, Sommer RJ, Aroian RV, Ghosh P (2012) Structure and glycolipid binding properties of the nematicidal protein Cry5B. Biochemistry 51(49):9911-9921. https://doi.org/10.1021/bi301386q

Kelker MS, Berry C, Evans SL, Pai R, McCaskill DG, Wang NX, Russell JC, Baker MD, Yang C, Pflugrath JW, Wade M (2014) Structural and biophysical characterization of Bacillus thuringiensis insecticidal proteins Cry34Ab1 and Cry35Ab1. PLoS One 9(11):e112555. https://doi.org/10.1371/journal.pone.0112555

Kumar S, Stecher G, Li M, Knyaz C, Tamura K (2018) MEGA X: molecular evolutionary genetics analysis across computing platforms. Mol Biol Evol 35(6):1547-1549. https://doi.org/10.1093/molbev/msy096

Li J, Carroll J, Ellar DJ (1991) Crystal structure of insecticidal $\delta$-endotoxin from Bacillus thuringiensisat $2.5 \AA$ resolution. Nature 353(6347):815-821. https://doi. org/10.1038/353815a0

López-Pazos SA, Gómez JE, Salamanca JA (2009) Cry1B and Cry3A are active against Hypothenemus hampei Ferrari (coleoptera: scolytidae). J Invertebr Pathol 101(3):242-245

McNall RJ, Adang MJ (2003) Identification of novel Bacillus thuringiensis Cry1Ac binding proteins in Manduca sexta midgut through proteomic analysis. Insect Biochem Mol Biol 33(10):999-1010. https://doi.org/10.1016/S0965-174 8(03)00114-0

Mizuki E, Park YS, Saitoh H, Yamashita S, Akao T, Higuchi K, Ohba M (2000) Parasporin, a human leukemic cell-recognizing parasporal protein of Bacillus thuringiensis. Clin Diagn Lab Immunol 7(4):625-634. https://doi.org/10.1128/ CDLI.7.4.625-634.2000

Morse RJ, Yamamoto T, Stroud RM (2001) Structure of Cry2Aa suggests an unexpected receptor binding epitope. Structure 9(5):409-417. https://doi. org/10.1016/S0969-2126(01)00601-3

Naimov S, Boncheva R, Karlova R, Dukiandjiev S, Minkov I, de Maagd RA (2008) Solubilization, activation, and insecticidal activity of Bacillus thuringiensis serovarthompsoni HD542 crystal proteins. Appl Environ Microbiol 74(23): 7145-7151. https://doi.org/10.1128/AEM.00752-08

Palma L, Muñoz D, Berry C, Murillo J, Caballero P (2014) Bacillus thuringiensis toxins: an overview of their biocidal activity. Toxins 6(12):3296-3325. https:// doi.org/10.3390/toxins6123296

Pigott CR, Ellar DJ (2007) Role of receptors in Bacillus thuringiensis crystal toxin activity. Microbiol Mol Biol Rev 71(2):255-281. https://doi.org/10.1128/MMBR. 00034-06

Raymond B, Johnston PR, Nielsen-LeRoux C, Lereclus D, Crickmore N (2010) Bacillus thuringiensis: an impotent pathogen? Trends Microbiol 18(5):189-194. https://doi.org/10.1016/j.tim.2010.02.006

Saitou N, Nei M (1987) The neighbor-joining method: a new method for reconstructing phylogenetic trees. Molecular biology and evolution 4(4):406425. https://doi.org/10.1093/oxfordjournals.molbev.a040454

Sanchis V, Bourguet D (2008) Bacillus thuringiensis: applications in agriculture and insect resistance management. A review. Agronom Sustain Devs 28(1):11-20. https://doi.org/10.1051/agro:2007054

Schnepf E, Crickmore NV, Van Rie J, Lereclus D, Baum J, Feitelson J, Zeigler DR, Dean D (1998) Bacillus thuringiensis and its pesticidal crystal proteins. Microbiol Mol Biol Rev 62(3):775-806. https://doi.org/10.1128/MMBR.62.3.775806.1998

Schnepf HE (1995) Bacillus thuringiensis toxins: regulation, activities and structural diversity. Curr Opin Biotechnol 6(3):305-312. https://doi.org/10.1016/0958-1 669(95)80052-2
Soberon M, Gill SS, Bravo A (2009) Signaling versus punching hole: How do Bacillus thuringiensis toxins kill insect midgut cells? Cell Mol Life Sci 66(8): 1337-1349. https://doi.org/10.1007/s00018-00-8330-9

Van Frankenhuyzen K (2009) Insecticidal activity of Bacillus thuringiensis crystal proteins. J Invertebr Pathol 101(1):1-6. https://doi.org/10.1016/j.jip.2009.02.009

Wang C, Li W, Kessenich CR, Petrick JS, Rydel TJ, Sturman EJ, Lee TC, Glenn KC, Edrington TC (2018) Safety of the Bacillus thuringiensis-derived Cry1A. 105 protein: evidence that domain exchange preserves mode of action and safety. Regul Toxicol Pharmacol 99:50-60. https://doi.org/10.1016/j.yrtph.2018. 09.003

Wang Y, Wang J, Fu X, Nageotte JR, Silverman J, Bretsnyder EC, Chen D, Rydel TJ, Bean GJ, Li KS, Kraft E (2019) Novel receptor interaction of Bacillus thuringiensis Cry1Da_7 and Cry1B. 868 proteins allow control of resistant fall armyworm, Spodoptera frugiperda (JE Smith). Appl Environ Microbiol AEM00579. https://doi.org/10.1128/AEM.00579-19

Zuckerkandl E, Pauling L (1965) Evolutionary divergence and convergence in proteins. Evolving Genes Proteins 1965:97-166. https://doi.org/10.1016/ B978-1-4832-2734-4.50017-6

\section{Publisher's Note}

Springer Nature remains neutral with regard to jurisdictional claims in published maps and institutional affiliations.

\section{Submit your manuscript to a SpringerOpen ${ }^{\circ}$ journal and benefit from:}

- Convenient online submission

- Rigorous peer review

- Open access: articles freely available online

- High visibility within the field

- Retaining the copyright to your article

Submit your next manuscript at $\boldsymbol{\nabla}$ springeropen.com 